\title{
Locally determined functions and Alperin's conjecture
}

\author{
Jacques Thévenaz \\ Institut de Mathématiques \\ Université de Lausanne \\ CH-1015 Lausanne \\ Switzerland
}

Nos codes, nos ambitions, notre politique sont inspirés de notions fortement, puissamment locales.

(Paul Valéry)

For a fixed prime number $p$, Alperin's conjecture asserts essentially that the function $k(G)-z(G)$ should be $p$-locally determined, where $k(G)$ is the number of absolutely irreducible representations of a finite group $G$ in characteristic zero, and $z(G)$ is the number of those representations whose dimension is a multiple of $|G|_{p}$, the $p$-part of the order of the group. Equivalently, the conjecture also says that the function $\ell(G)-z(G)$ should be $p$-locally determined, where $\ell(G)$ is this time the number of absolutely irreducible representations of $G$ in characteristic $p$, and $z(G)$ is the number of those representations which are both simple and projective modules over the group algebra, in other words the number of blocks of $G$ of defect zero (this number $z(G)$ is well-known to be equal to the one defined above).

We first consider the question of a precise mathematical definition of $p$-local determination. We give a combinatorial definition involving all subgroups of $G$ and procede to show that any function $f$ on the poset of subgroups of $G$, which is constant on conjugacy classes of subgroups, decomposes uniquely as the sum of two functions $f=f_{p}+f_{p^{\prime}}$ where $f_{p}$ is $p$-locally determined and $f_{p^{\prime}}$ vanishes on $p$-local subgroups (where by definition, any subgroup $H$ such that $O_{p}(H) \neq 1$ is called a $p$-local subgroup). This can be viewed as a decomposition of functions into a $p$-part and a $p^{\prime}$-part. For instance the $p$-part of the constant function $f=1$ is the characteristic function of subgroups of order divisible by $p$. We have various results on $p$-locally determined functions which involve the simplicial complex of non-trivial $p$-subgroups introduced by K.S. Brown. In particular there are explicit formulae for finding the $p$-part and the $p^{\prime}$-part of a function, which find their origin in the work of R. Knörr and G. Robinson $[\mathrm{KR}]$.

It is well-known that the above function $z$ vanishes on $p$-local subgroups and it turns out that Alperin's conjecture is equivalent to the assertion that the function $k-z$ is the $p$-part of the function $k$ (and similarly with the function $\ell$ ). This can be expressed in a combinatorial fashion which provides new versions of Alperin's conjecture, as follows.

CONJECTURE 1. If one writes $k(H)-z(H)=\frac{1}{|H|} \sum_{S \leq H} f_{1}(S)$ for all $H \leq G$, then the (uniquely determined) function $f_{1}$ vanishes on subgroups $H$ such that $O_{p}(H)=1$. 
CONJECTURE 2. If one writes $\ell(H)-z(H)=\frac{1}{|H|} \sum_{S \leq H} f_{2}(S)$ for all $H \leq G$, then the (uniquely determined) function $f_{2}$ vanishes on subgroups $H$ such that $O_{p}(H)=1$.

Similarly there is another combinatorial version of the conjecture involving the function $z$. In order to state this conjecture, we let $\mathcal{A}$ be the family of subgroups $S$ of $G$ such that $O_{p}(S)$ is an elementary abelian $p$-group and $S / O_{p}(S)$ is the direct product of (at most) two cyclic $p^{\prime}$-groups.

CONJECTURE 3. If one writes $z(H)=\frac{1}{|H|} \sum_{S \leq H} \widehat{z}(S)$ for all $H \leq G$, then the (uniquely determined) function $\widehat{z}$ vanishes on subgroups $H$ which are not in $\mathcal{A}$.

Finally our explicit formulae for finding the $p$-part and the $p^{\prime}$-part of a function allow to make computations. We obtain in this way an explicit conjectural formula for $z(G)$ which again is equivalent to Alperin's conjecture. For $S \in \mathcal{A}$, we write $\mu\left(\left(1, O_{p}(S)\right)^{S}\right)$ for the Möbius function of the poset of $S$-stable subgroups of $O_{p}(S)$ (i.e. normal in $S$ ) and we let $\phi_{2}\left(S / O_{p}(S)\right.$ ) be the number of pairs of (commuting) elements which generate $S / O_{p}(S)$.

CONJECTURE 4 . The number $z(G)$ is given by the formula

$$
z(G)=\frac{1}{|G|} \sum_{S \in \mathcal{A}} \mu\left(\left(1, O_{p}(S)\right)^{S}\right)\left|O_{p}(S): C_{O_{p}(S)}(S)\right| \phi_{2}\left(S / O_{p}(S)\right) .
$$

Thus the conjecture gives a solution to Brauer's problem of describing $z(G)$ directly in terms of the group structure. Of course there is already a solution to this problem due to Robinson [Ro] (and which is proved!), but the conjecture here is a formula of a completely different nature. It should be noted that it is often easy to compute the right hand side: one essentially needs to know which subgroups of $G$ lie in $\mathcal{A}$, because the various integers appearing in the formula are easy to handle.

There is a block-theoretic version of Alperin's conjecture which is stronger than the overall statement which we are considering in this paper. Unfortunately, because of its combinatorial nature involving all subgroups, our approach has no obvious generalization to the case of blocks. Hopefully this situation will be improved later. Meanwhile, as mentioned above, we view the overall approach to Alperin's conjecture as a solution to Brauer's problem.

\section{Inversion of functions}

Let $S(G)$ be the poset of all subgroups of a finite group $G$ and let $A$ be an abelian group. Given a function $f: S(G) \rightarrow A$, there is a unique function $\widehat{f}: S(G) \rightarrow A$ such that

$$
|H| f(H)=\sum_{S \leq H} \widehat{f}(S) \quad \text { for all } H \in S(G) .
$$

By Möbius inversion, (1.1) is equivalent to the following equation which gives a formula for $\widehat{f}$ and shows its uniqueness:

$$
\widehat{f}(H)=\sum_{S \leq H} \mu(S, H)|S| f(S) \quad \text { for all } H \in S(G),
$$


where $\mu$ denotes the Möbius function of the poset $S(G)$. One could of course also invert functions without multiplying by the order of the group, but that would not give the "natural" definition: there is an interpretation of the subsequent results in the Burnside ring which gives a mathematical meaning to this naturality (see Section 7). Note that if $A=\mathbb{Z}$, then for each $f$, the function $\widehat{f}$ is integral valued. However given an integral valued function $\widehat{f}$, the corresponding $f$ has values in $\mathbb{Q}$ in general.

We shall only work with functions which are constant on conjugacy classes of subgroups (but we need the full poset $S(G)$ for their inversion). We define $\mathcal{F}(G, A)$ to be the set of all functions $f: S(G) \rightarrow A$ such that $f\left(g H g^{-1}\right)=f(H)$ for all $g \in G$ and $H \in S(G)$. This is clearly an abelian group.

Let $\mathcal{X}$ be a set of subgroups of $G$ (closed under conjugation). A function $f \in \mathcal{F}(G, A)$ will be called $\mathcal{X}$-determined if $\widehat{f}(H)=0$ for $H \notin \mathcal{X}$. Thus by (1.1) the function $f$ can be written as a sum running only over subgroups in the class $\mathcal{X}$.

The usual definition of a $p$-local subgroup of a finite group $G$ (namely the normalizer of a non-trivial $p$-subgroup) depends on the way the subgroup is embedded in $G$. We extend this definition to obtain an intrinsic notion: a finite group $H$ is called $p$-local if there exists a non-trivial normal $p$-subgroup in $H$. Thus a $p$-local subgroup $H$ of $G$ is now any subgroup sandwiched between a non-trivial $p$-subgroup $P$ and its normalizer. A function $f \in \mathcal{F}(G, A)$ is called $p$-locally determined if it is $\mathcal{X}$-determined where $\mathcal{X}$ is the set of p-local subgroups of $G$. For another approach, see Section 5 .

Our main interest is in the class of $p$-local subgroups but we first give some other examples.

EXAMPLES. In all the following examples, the abelian group $A$ is the field $\mathbb{Q}$ of rational numbers (or $\mathbb{Z}$ when no denominators are involved).

(1.3) The function $f(H)=|H|^{-1}$ is $\{1\}$-determined. We have $\widehat{f}(1)=1$ and $\widehat{f}(H)=0$ if $H \neq 1$.

(1.4) More generally if $K$ is a subgroup of $G$ and $\mathcal{X}_{K}$ is the set of conjugates of $K$, then taking $\widehat{f}$ to be the characteristic function of the set $\mathcal{X}_{K}$, we see that the following function is $\mathcal{X}_{K}$-determined:

$$
f(H)= \begin{cases}|H|^{-1} m(K, H) & \text { if } K \leq_{G} H, \\ 0 & \text { otherwise }\end{cases}
$$

where $m(K, H)$ is the number of $G$-conjugates of $K$ contained in $H$.

(1.5) The constant function $f=1$ is $\mathcal{C}$-determined where $\mathcal{C}$ is the family of all cyclic subgroups of $G$. On a cyclic group $C$, the function $\widehat{f}$ takes the value $\widehat{f}(C)=\phi(|C|)$ where $\phi$ is the Euler function (i.e. the number of generators of $C$ ).

(1.6) Our next example is more or less well known. It is for instance implicit in the work of Hirsch [Hi]. Take $k(H)$ to be the number of conjugacy classes of elements of $H$, that is also the number of absolutely irreducible representations of $H$ over $\mathbb{C}$. Then we have

$$
\begin{aligned}
|H| k(H) & =|H| \sum_{\text {classes }} 1=|H| \sum_{x \in H}\left|H: C_{H}(x)\right|^{-1} \\
& =\sum_{x \in H}\left|C_{H}(x)\right|=\sum_{\substack{x, y \in H \\
x y=y x}} 1 \\
& =\sum_{S \leq H} \phi_{2}(S)
\end{aligned}
$$

where $\phi_{2}(S)$ is the number of commuting pairs $(x, y)$ such that $S=<x, y>$. Thus $\phi_{2}(S)=0$ if $S$ is not the direct product of two cyclic groups. The above computation shows that $\widehat{k}=\phi_{2}$ so that $k$ is $\mathcal{C}_{2}$-determined, where $\mathcal{C}_{2}$ is the family of subgroups which are direct products of two cyclic groups. It is not 
difficult to give formulae for the function $\phi_{2}$. If $q$ is a prime and $C_{q^{n}}$ denotes the cyclic group of prime power order $q^{n}$, then for $n, m \geq 1$ we have

$$
\phi_{2}\left(C_{q^{n}}\right)=q^{2 n-2}\left(q^{2}-1\right), \quad \phi_{2}\left(C_{q^{n}} \times C_{q^{m}}\right)=q^{2 n+2 m-3}\left(q^{2}-1\right)(q-1) .
$$

This suffices to determine entirely $\phi_{2}$ thanks to the property $\phi_{2}(H \times K)=\phi_{2}(H) \phi_{2}(K)$ if $H$ and $K$ have coprime orders.

(1.8) Given a prime $p$, there is a similar treatment for the function $\ell(H)$, defined to be the number of conjugacy classes of elements of $H$ of order prime to $p$, that is also the number of absolutely irreducible representations of $H$ over a field of characteristic $p$. In the computation of the previous example, the sum now runs only over elements $x \in H$ of order prime to $p$ (but $y$ is arbitrary). It follows that $\ell$ is determined by the class of subgroups which are direct product of two cyclic groups, one of which being of order prime to $p$. There are also explicit formulae for the function $\widehat{\ell}$. If $q$ is a prime different from $p$, then

$$
\widehat{\ell}\left(C_{q^{n}}\right)=\phi_{2}\left(C_{q^{n}}\right), \quad \widehat{\ell}\left(C_{q^{n}} \times C_{q^{m}}\right)=\phi_{2}\left(C_{q^{n}} \times C_{q^{m}}\right),
$$

which are given by (1.7). For the prime $p$, the formulae are

$$
\widehat{\ell}\left(C_{p^{n}}\right)=\phi\left(p^{n}\right)=p^{n-1}(p-1), \quad \widehat{\ell}\left(C_{p^{n}} \times C_{p^{m}}\right)=0 .
$$

Note that if $H$ is a group of order prime to $p$, then $\ell(H)=k(H)$ and $\widehat{\ell}(H)=\widehat{k}(H)$.

\section{Preliminaries on Brown's complex}

Let us first recall the definition of Brown's simplicial complex for a finite group $H$. Given a prime $p$, let $S_{p}(H)$ be the poset of all non-trivial $p$-subgroups of $H$. Brown's complex $\Delta\left(S_{p}(H)\right)$ is the simplicial complex whose simplices are the totally ordered subsets of $S_{p}(H)$, including the empty subset which we view as a simplex of dimension -1 . Thus an $n$-simplex $\sigma$ is a chain $P_{0}<P_{1}<\ldots<P_{n}$ in $S_{p}(H)$ and its dimension $n$ is written $n=\operatorname{dim}(\sigma)$.

The group $H$ acts by conjugation on $S_{p}(H)$, hence also on $\Delta\left(S_{p}(H)\right)$. We denote by $\left[\Delta\left(S_{p}(H)\right) / H\right]$ an arbitrary set of representatives of $H$-orbits in $\Delta\left(S_{p}(H)\right)$. For each simplex $\sigma \in \Delta\left(S_{p}(H)\right)$, the stabilizer $H_{\sigma}$ of $\sigma$ is the intersection of the normalizers of the $p$-subgroups in $\sigma$. Since the smallest element $P_{0}$ of the chain $\sigma$ is a normal subgroup of $H_{\sigma}$, the subgroup $H_{\sigma}$ is always a $p$-local subgroup of $H$, except when $\sigma=\emptyset$ where $H_{\sigma}=H$.

We need several results involving $\Delta\left(S_{p}(H)\right)$ and functions defined on subgroups. We shall adopt a conceptual approach (based on [Th] and [W2]) using easy simplicial topology and the Burnside ring. But we shall also indicate a second proof of each result using elementary counting arguments.

Let $B(H)$ be the Burnside ring of $H$. If $\Delta$ is a finite simplicial $H$-complex, the set $\Delta_{k}$ of $k$-simplices of $\Delta$ is an $H$-set, thus an element of $B(H)$. The alternating sum

$$
\widetilde{\Lambda}_{H}(\Delta)=\sum_{i=-1}^{\operatorname{dim}(\Delta)}(-1)^{i} \Delta_{i}=\sum_{\sigma \in[\Delta / H]}(-1)^{\operatorname{dim}(\sigma)} H / H_{\sigma}
$$

is an element of $B(H)$ called the reduced Lefschetz $H$-set (or reduced Lefschetz invariant) of $\Delta$. This is an invariant of $H$-homotopy type which was studied in [Th]. 
Following Webb [W2, §6], we can view any function $f \in \mathcal{F}(H, A)$ as a $\mathbb{Z}$-linear map $f: B(H) \rightarrow A$ by defining $f(H / S)=f(S)$ for any subgroup $S \leq H$, and extending $\mathbb{Z}$-linearly. Since $f$ is constant on conjugacy classes of subgroups, this is well defined. Thus we can apply $f$ to $\widetilde{\Lambda}_{H}(\Delta)$ and get

$$
f\left(\widetilde{\Lambda}_{H}(\Delta)\right)=\sum_{\sigma \in[\Delta / H]}(-1)^{\operatorname{dim}(\sigma)} f\left(H_{\sigma}\right) .
$$

For dealing with such a sum, it suffices to have information about the $H$-homotopy type of $\Delta$. For instance in order to prove the vanishing of such a sum, it suffices to show that $\Delta$ is $H$-contractible, since it has zero reduced Lefschetz $H$-set in that case [Th]. Thus in such a situation, we see immediately that one obtains zero whatever the function $f$ is.

(2.1) LEMMA. If $H$ is a $p$-local group, then for each $f \in \mathcal{F}(H, A)$, we have

$$
\sum_{\sigma \in\left[\Delta\left(S_{p}(H)\right) / H\right]}(-1)^{\operatorname{dim}(\sigma)} f\left(H_{\sigma}\right)=0 .
$$

First proof. Quillen $[\mathrm{Qu}]$ proved that $\Delta\left(S_{p}(H)\right)$ is contractible when $H$ is $p$-local. The contraction given by Quillen is in fact $H$-equivariant, so $\Delta\left(S_{p}(H)\right)$ is $H$-contractible. Therefore $f\left(\widetilde{\Lambda}_{H}\left(\Delta\left(S_{p}(H)\right)\right)\right)=$ 0 .

Second proof. Using the argument of [KR, 4.1], the chains in $\Delta\left(S_{p}(H)\right)$ can be paired so that the alternating sum is zero.

(2.2) LEMMA. Let $S$ be a $p$-local subgroup of $H$ and let $S_{p}(H)^{S}$ be the poset of $S$-invariant non-trivial $p$-subgroups of $H$. For every subgroup $X$ of $N_{H}(S)$ and for every $f \in \mathcal{F}(X, A)$, we have

$$
\sum_{\sigma \in\left[\Delta\left(S_{p}(H)^{S}\right) / X\right]}(-1)^{\operatorname{dim}(\sigma)} f\left(X_{\sigma}\right)=0 .
$$

First proof. $\Delta\left(S_{p}(H)^{S}\right)$ is $N_{H}(S)$-contractible (hence $X$-contractible) by the contraction

$$
P \mapsto P \cdot O_{p}(S) \mapsto O_{p}(S) \quad\left(P \in \Delta\left(S_{p}(H)^{S}\right)\right)
$$

(see $[\mathrm{TW}, 1.2]$ for more details). Therefore $f\left(\widetilde{\Lambda}_{X}\left(\Delta\left(S_{p}(H)^{S}\right)\right)\right)=0$.

Second proof. Using again the argument of [KR, 4.1], the chains in $\Delta\left(S_{p}(H)^{S}\right)$ can be paired so that the alternating sum is zero.

(2.3) LEMMA. Let $P \in S_{p}(H)$, and let $S_{p}(H)_{>P}=\left\{Q \in S_{p}(H) \mid Q>P\right\}$. Consider the action of $N_{H}(P)$ on both $S_{p}(H)_{>P}$ and $S_{p}\left(N_{H}(P) / P\right)$. Then for each $f \in \mathcal{F}\left(N_{H}(P), A\right)$, we have

$$
\begin{aligned}
\sum_{\sigma \in\left[\Delta\left(S_{p}(H)>P\right) / N_{H}(P)\right]}(-1)^{\operatorname{dim}(\sigma)} & f\left(N_{H}(P)_{\sigma}\right) \\
= & \sum_{\sigma \in\left[\Delta\left(S_{p}\left(N_{H}(P) / P\right)\right) / N_{H}(P)\right]}(-1)^{\operatorname{dim}(\sigma)} f\left(N_{H}(P)_{\sigma}\right) .
\end{aligned}
$$

First proof. $S_{p}\left(N_{H}(P) / P\right)$ is $N_{H}(P)$-homotopy equivalent to $S_{p}(H)_{>P}$ by [W2, 6.4]. Therefore $f\left(\widetilde{\Lambda}_{N_{H}(P)}\left(\Delta\left(S_{p}(H)_{>P}\right)\right)\right)=f\left(\widetilde{\Lambda}_{N_{H}(P)}\left(\Delta\left(S_{p}\left(N_{H}(P) / P\right)\right)\right)\right)$.

Second proof. Using the argument of the first part of the proof of (3.3) in $[\mathrm{KR}]$, the chains in $\Delta\left(S_{p}(H)_{>P}\right)-\Delta\left(S_{p}\left(N_{H}(P)\right)_{>P}\right)$ can be paired so that their contribution to the alternating sum is zero.

Although we do not need the following result in this paper, we mention it for completeness. 
(2.4) LEMMA. Let $A_{p}(H)$ be the poset of non-trivial elementary abelian $p$-subgroups of $H$. Let $B_{p}(H)$ be the poset of non-trivial $p$-subgroups $P$ of $H$ such that $P=O_{p}\left(N_{H}(P)\right)$. Let $R_{p}(H)$ be the subcomplex of $\Delta\left(S_{p}(H)\right)$ consisting of the chains whose maximal element normalizes all the other subgroups in the chain. Then for each $f \in \mathcal{F}(H, A)$, we have

$$
\begin{aligned}
\sum_{\sigma \in\left[\Delta\left(S_{p}(H)\right) / H\right]}(-1)^{\operatorname{dim}(\sigma)} f\left(H_{\sigma}\right) & =\sum_{\sigma \in\left[\Delta\left(A_{p}(H)\right) / H\right]}(-1)^{\operatorname{dim}(\sigma)} f\left(H_{\sigma}\right) \\
& =\sum_{\sigma \in\left[\Delta\left(B_{p}(H)\right) / H\right]}(-1)^{\operatorname{dim}(\sigma)} f\left(H_{\sigma}\right) \\
& =\sum_{\sigma \in\left[R_{p}(H) / H\right]}(-1)^{\operatorname{dim}(\sigma)} f\left(H_{\sigma}\right) .
\end{aligned}
$$

First proof. $\Delta\left(S_{p}(H)\right), \Delta\left(A_{p}(H)\right), \Delta\left(B_{p}(H)\right)$ and $R_{p}(H)$ are $H$-homotopy equivalent by [TW, Theorem 2]. Therefore

$$
f\left(\widetilde{\Lambda}_{H}\left(\Delta\left(S_{p}(H)\right)\right)\right)=f\left(\widetilde{\Lambda}_{H}\left(\Delta\left(A_{p}(H)\right)\right)\right)=f\left(\widetilde{\Lambda}_{H}\left(\Delta\left(B_{p}(H)\right)\right)\right)=f\left(\widetilde{\Lambda}_{H}\left(R_{p}(H)\right)\right) .
$$

Second proof. This is exactly [KR, 3.3].

\section{Decomposition of functions}

Let $\mathcal{F}(G, A)$ be the set of all functions $f: S(G) \rightarrow A$ which are constant on conjugacy classes of subgroups. We define two subsets of $\mathcal{F}(G, A)$.

$$
\begin{aligned}
\mathcal{F}_{p}(G, A) & =\left\{f \in \mathcal{F}(G, A) \mid \widehat{f}(H)=0 \text { if } O_{p}(H)=1\right\}, \\
\mathcal{F}_{p^{\prime}}(G, A) & =\left\{f \in \mathcal{F}(G, A) \mid f(H)=0 \text { if } O_{p}(H) \neq 1\right\} .
\end{aligned}
$$

Thus $\mathcal{F}_{p}(G, A)$ is the set of $p$-locally determined functions while $\mathcal{F}_{p^{\prime}}(G, A)$ is the set of functions which vanish on $p$-local subgroups. It is clear that $\mathcal{F}(G, A)$ is an abelian group and that both $\mathcal{F}_{p}(G, A)$ and $\mathcal{F}_{p^{\prime}}(G, A)$ are subgroups of $\mathcal{F}(G, A)$. Our main result is the following.

(3.1) THEOREM. $\quad \mathcal{F}(G, A)=\mathcal{F}_{p}(G, A) \oplus \mathcal{F}_{p^{\prime}}(G, A)$.

The main ingredient for the proof of the theorem is an explicit formula for the projection onto each factor of the direct sum. This involves Brown's simplicial complex. We define two $\mathbb{Z}$-linear maps

$$
\begin{aligned}
\mathcal{F}(G, A) & \rightarrow \mathcal{F}(G, A) \quad ; \quad f_{p}(H)=\sum_{\sigma \in\left[\Delta\left(S_{p}(H)\right) / H\right]}(-1)^{\operatorname{dim}(\sigma)} f\left(H_{\sigma}\right), \\
f & \mapsto f_{p} \\
\mathcal{F}(G, A) & \rightarrow \mathcal{F}(G, A) \quad ; \quad f_{p^{\prime}}(H)=\sum_{\sigma \in\left[\Delta\left(S_{p}(H)\right) / H\right]}(-1)^{\operatorname{dim}(\sigma)+1} f\left(H_{\sigma}\right) . \\
f & \mapsto f_{p^{\prime}}
\end{aligned}
$$

Notice that by Lemma 2.4, we can also define $f_{p}$ and $f_{p^{\prime}}$ using any of the other simplicial complexes defined in Lemma 2.4. This remark also applies throughout this paper whenever we use Brown's complex.

Our aim is to show that the two maps above are the projections onto $\mathcal{F}_{p}(G, A)$ and $\mathcal{F}_{p^{\prime}}(G, A)$ respectively. First note that their sum is the identity because there is only the term for $\sigma=\emptyset$ left in the sum $f_{p}(H)+f_{p^{\prime}}(H)$, and $H_{\emptyset}=H$. Next we show that the second map is indeed the projection onto $\mathcal{F}_{p^{\prime}}(G, A)$. 
(3.2) PROPOSITION. Let $f \in \mathcal{F}(G, A)$.

(a) $f_{p^{\prime}} \in \mathcal{F}_{p^{\prime}}(G, A)$.

(b) If $f \in \mathcal{F}_{p^{\prime}}(G, A)$, then $f=f_{p^{\prime}}$.

Proof. (a) One has to show that $f_{p^{\prime}}$ vanishes on $p$-local subgroups. This is exactly Lemma 2.1 .

(b) Since every stabilizer $H_{\sigma}$ of a non-empty simplex $\sigma \in \Delta\left(S_{p}(H)\right)$ is a $p$-local subgroup, the function $f$ vanishes on those subgroups. Thus the only term remaining in the sum which defines $f_{p^{\prime}}$ corresponds to the empty simplex and we get $f_{p^{\prime}}(H)=f(H)$.

In order to show that the map $f \mapsto f_{p}$ is the projection onto $\mathcal{F}_{p}(G, A)$, we need information about the inversion of $f_{p}$, or equivalently, of $f_{p^{\prime}}$.

(3.3) PROPOSITION. Let $f \in \mathcal{F}(G, A)$. Then the inversion of $f_{p^{\prime}}$ is given by the formula

$$
\widehat{f}_{p^{\prime}}(H)=\mu\left(\left(1, O_{p}(H)\right)^{H}\right) \sum_{S \in O_{p}(H)^{\perp}} \widehat{f}(S)
$$

where $O_{p}(H)^{\perp}$ denotes the set of complements of $O_{p}(H)$ in $H$ and $\mu\left(\left(1, O_{p}(H)\right)^{H}\right)$ is the Möbius function of the poset $\left(1, O_{p}(H)\right)^{H}$ of $H$-invariant subgroups of $O_{p}(H)$ (i.e. normal in $H$ ).

Note that $\mu\left(\left(1, O_{p}(H)\right)^{H}\right)=0$ unless $O_{p}(H)$ is elementary abelian and the action of $H / O_{p}(H)$ on $O_{p}(H)$ is semi-simple. Indeed otherwise the intersection of all maximal $H$-stable subgroups of $O_{p}(H)$ is non-trivial and this is well known to imply the vanishing of the Möbius function [St, 3.9.5].

Note also that if $f$ is constant on isomorphism classes of subgroups (this is often the case), then the value $\widehat{f}(S)$ is constant and there is not any more sum. Although the function $f$ (hence also $\widehat{f}$ ) is only defined on subgroups of $H$, one can define $f$ and $\widehat{f}$ on subgroups of $H / O_{p}(H)$ via the isomorphism $S \cong H / O_{p}(H)$ (where $S \in O_{p}(H)^{\perp}$ is an arbitrary complement), and this leads to the formula

$$
\widehat{f_{p^{\prime}}}(H)=\left|O_{p}(H)^{\perp}\right| \mu\left(\left(1, O_{p}(H)\right)^{H}\right) \widehat{f}\left(H / O_{p}(H)\right),
$$

which holds when $f$ is constant on isomorphism classes of subgroups.

Assuming that $O_{p}(H)$ is abelian (as we may by the remark above), we now consider the special case where all complements of $O_{p}(H)$ are conjugate in $H$ (e.g. when $H / O_{p}(H)$ and $O_{p}(H)$ have coprime orders, that is when $H / O_{p}(H)$ is a $p^{\prime}$-group). Since $\widehat{f}$ is constant on conjugacy classes of subgroups, again there is no sum. Moreover $O_{p}(H)$ acts transitively on $O_{p}(H)^{\perp}$ and the stabilizer of one complement $S$ is $C_{O_{p}(H)}(S)$ (because it is $N_{H}(S) \cap O_{p}(H)$ which normalizes $S$ and is normalized by $S$, hence centralizes $S)$. But since $O_{p}(H)$ is abelian and $H=O_{p}(H) S$, this stabilizer is also $C_{O_{p}(H)}(H)$ and therefore the number of complements is $\left|O_{p}(H)^{\perp}\right|=\left|O_{p}(H): C_{O_{p}(H)}(H)\right|$. Thus we have proved:

(3.5) COROLLARY. With the notation of Proposition 3.3, if moreover all complements of $O_{p}(H)$ in $H$ are conjugate, then

$$
\widehat{f_{p^{\prime}}}(H)=\left|O_{p}(H): C_{O_{p}(H)}(H)\right| \mu\left(\left(1, O_{p}(H)\right)^{H}\right) \widehat{f}\left(H / O_{p}(H)\right) .
$$

Another consequence of Proposition 3.3 is the following. 
(3.6) COROLLARY. If $f$ is $\mathcal{X}$-determined, then $f_{p^{\prime}}$ is $\mathcal{X}(p)$-determined, where $\mathcal{X}(p)$ is the family of all subgroups $H$ such that $H / O_{p}(H) \in \mathcal{X}, O_{p}(H)$ is elementary abelian, the action of $H / O_{p}(H)$ on $O_{p}(H)$ is semi-simple and $O_{p}(H)$ has a complement in $H$.

Proof. If $\widehat{f}_{p^{\prime}}(H) \neq 0$, then the formula of Proposition 3.3 implies that $O_{p}(H)^{\perp}$ is non-empty, $\widehat{f}(S) \neq 0$ for some $S \in O_{p}(H)^{\perp}$ so that $H / O_{p}(H) \cong S$ belongs to $\mathcal{X}$, and finally $\mu\left(\left(1, O_{p}(H)\right)^{H}\right) \neq 0$. As noted above, this last inequality implies that $O_{p}(H)$ is elementary abelian and that the action of $H / O_{p}(H)$ on $O_{p}(H)$ is semi-simple.

Once Theorem 3.1 is established, Corollary 3.6 also implies that if $f$ is $\mathcal{X}$-determined, then $f_{p}$ is $\mathcal{Y}$ determined where $\mathcal{Y}=\mathcal{X} \cup \mathcal{X}(p)$. Moreover one can throw away from $\mathcal{Y}$ all subgroups $H$ with $O_{p}(H)=1$, by definition of $p$-locally determined functions.

Now we come to the proof of Proposition 3.3, which is the crucial step for Theorem 3.1.

Proof of Proposition 3.3. Recall [St, 3.8.6] that the Möbius function of a poset $\mathcal{X}$ with a minimal element $x_{0}$ and the reduced Euler characteristic of the associated simplicial complex $\Delta\left(\mathcal{X}-\left\{x_{0}\right\}\right)$ are related by the formula

$$
\tilde{\chi}\left(\Delta\left(\mathcal{X}-\left\{x_{0}\right\}\right)=-\sum_{x \in \mathcal{X}} \mu\left(x_{0}, x\right) .\right.
$$

We shall apply this to the complex $\Delta^{S}$ of $S$-stable simplices in $\Delta=\Delta\left(S_{p}(H)\right)$; here $\mathcal{X}=S_{p}(H)^{S} \cup\{1\}$. Note that if $O_{p}(S) \neq 1$, then by Lemma 2.2 applied to the constant function 1, we have

$$
\widetilde{\chi}\left(\Delta^{S}\right)=\sum_{\sigma \in\left[\Delta^{S} / N_{H}(S)\right]}(-1)^{\operatorname{dim}(\sigma)}=0 .
$$

Now we can start the calculation.

$$
\begin{aligned}
|H| f_{p^{\prime}}(H) & =\sum_{\sigma \in \Delta}|H|\left|H: H_{\sigma}\right|^{-1}(-1)^{\operatorname{dim}(\sigma)+1} f\left(H_{\sigma}\right)=\sum_{\sigma \in \Delta}(-1)^{\operatorname{dim}(\sigma)+1} \sum_{S \leq H_{\sigma}} \widehat{f}(S) \\
& =\sum_{S \leq H} \widehat{f}(S) \sum_{\sigma \in \Delta^{S}}(-1)^{\operatorname{dim}(\sigma)+1}=\sum_{S \leq H} \widehat{f}(S)\left(-\widetilde{\chi}\left(\Delta^{S}\right)\right)
\end{aligned}
$$

and therefore, using (3.8),

$$
|H| f_{p^{\prime}}(H)=\sum_{S \leq H} \widehat{f}(S)\left(-\widetilde{\chi}\left(\Delta^{S}\right)\right)=\sum_{O_{p}(S)=1} \widehat{f}(S)\left(-\widetilde{\chi}\left(\Delta^{S}\right)\right) .
$$

Applying the formula (3.7), we get

$$
|H| f_{p^{\prime}}(H)=\sum_{O_{p}(S)=1} \widehat{f}(S) \sum_{P \in S_{p}(H)^{S} \cup\{1\}} \mu\left((1, P)^{S}\right)=\sum_{P \in S_{p}(H) \cup\{1\}} \sum_{\substack{S \leq N_{H}(P) \\ O_{p}(S)=1}} \widehat{f}(S) \mu\left((1, P)^{S}\right) .
$$

Note now that we necessarily have $S \cap P=1$ because $S \cap P \triangleleft S$ and $O_{p}(S)=1$. Therefore we can consider the subgroup $K=P S$ for which $O_{p}(K)=P$ and $S \in O_{p}(K)^{\perp}$. Thus we obtain

$$
|H| f_{p^{\prime}}(H)=\sum_{K} \sum_{S \in O_{p}(K)^{\perp}} \mu\left(\left(1, O_{p}(K)\right)^{S}\right) \widehat{f}(S)
$$

and it follows that

$$
\widehat{f}_{p^{\prime}}(K)=\sum_{S \in O_{p}(K)^{\perp}} \mu\left(\left(1, O_{p}(K)\right)^{S}\right) \widehat{f}(S) .
$$

If $O_{p}(K)$ is not (elementary) abelian, then $\mu\left(1, O_{p}(K)\right)^{S}=0$, while if it is abelian, then $\mu\left(\left(1, O_{p}(K)\right)^{S}\right)=$ $\mu\left(\left(1, O_{p}(K)\right)^{K}\right)$ since an $S$-stable subgroup of $O_{p}(K)$ is stable under $O_{p}(K) S=K$. Thus we deduce the required formula for $\widehat{f}_{p^{\prime}}(K)$. 
(3.10) REMARK. Since many functions $f$ project onto the same function $f_{p^{\prime}}$, the formula in Proposition 3.3 gives many possible expressions for $\widehat{f}_{p^{\prime}}$, depending on the choice of $f$. In particular we can choose $f=f_{p^{\prime}}$. For this special choice (i.e. when $f \in \mathcal{F}_{p^{\prime}}(G, A)$ ), we now sketch another proof of Proposition 3.3 based on the Crapo complementation formula for the Möbius function [Ai, 4.33]. Letting $P=O_{p}(K)$, we have by assumption $f(S)=0$ if $S \leq K$ and $S \cap P \neq 1$. So if we write $P S^{\perp}$ for the set of complements of $P S$ in the lattice $[S, K]$, we obtain

$$
\begin{aligned}
\widehat{f}(K) & =\sum_{S \leq K} \mu(S, K)|S| f(S)=\sum_{S \cap P=1} \mu(S, K)|S| f(S) \\
& =\sum_{S \cap P=1} \sum_{C \in P S^{\perp}} \mu(S, C) \mu(C, K)|S| f(S) \\
& =\sum_{C \in P^{\perp}} \mu(C, K) \sum_{S \leq C} \mu(S, C)|S| f(S)=\sum_{C \in P^{\perp}} \mu(C, K) \widehat{f}(C)
\end{aligned}
$$

and we conclude with the observation that $\mu(C, K)=\mu\left((1, P)^{K}\right)$ because the lattices $[C, K]$ and $[1, P]^{C}$ are isomorphic, and $[1, P]^{C}=[1, P]^{K}$ when $P$ is abelian (while $\mu\left((1, P)^{C}\right)=0$ if $P$ is not (elementary) abelian).

We can now finish the proof of Theorem 3.1 by showing that the map $f \mapsto f_{p}$ is indeed the projection onto $\mathcal{F}_{p}(G, A)$. Since we already know that $f=f_{p}+f_{p^{\prime}}$ for all $f$ and that $f \mapsto f_{p^{\prime}}$ is the projection onto $\mathcal{F}_{p^{\prime}}(G, A)$, this is equivalent to the following statement.

(3.11) PROPOSITION. Let $f \in \mathcal{F}(G, A)$. Then $f \in \mathcal{F}_{p}(G, A)$ if and only if $f_{p^{\prime}}=0$.

Proof. If $f_{p^{\prime}}=0$, then $\widehat{f}_{p^{\prime}}=0$ too. Thus if $H$ is such that $O_{p}(H)=1$, it follows from Proposition 3.3 that $0=\widehat{f}_{p^{\prime}}(H)=\widehat{f}(H)$. This proves that $f \in \mathcal{F}_{p}(G, A)$.

Assume now that $f \in \mathcal{F}_{p}(G, A)$. Applying Proposition 3.3 with an arbitrary subgroup $H$, we obtain

$$
\widehat{f}_{p^{\prime}}(H)=\mu\left(\left(1, O_{p}(H)\right)^{H}\right) \sum_{S \in O_{p}(H)^{\perp}} \widehat{f}(S)=0,
$$

because $\widehat{f}(S)=0$ since $O_{p}(S)=1$. Therefore the function $\widehat{f}_{p^{\prime}}$ is zero, hence $f_{p^{\prime}}=0$.

By Propositions 3.2 and 3.11, the proof of Theorem 3.1 is now complete.

\section{Some examples}

We give examples of the decomposition of functions, starting first with some easy functions which were already considered in Section 1.

(4.1) EXAMPLE. For the function $f(H)=|H|^{-1}$ of (1.3), we have

$$
\begin{aligned}
f_{p}(H) & =\sum_{\substack{\sigma \in\left[\Delta\left(S_{p}(H)\right) / H\right] \\
\sigma \neq \emptyset}}(-1)^{\operatorname{dim}(\sigma)}\left|H_{\sigma}\right|^{-1}=|H|^{-1} \sum_{\substack{\sigma \in \Delta\left(S_{p}(H)\right) \\
\sigma \neq \emptyset}}(-1)^{\operatorname{dim}(\sigma)} \\
& =|H|^{-1} \chi\left(\Delta\left(S_{p}(H)\right)\right.
\end{aligned}
$$


and similarly $f_{p^{\prime}}(H)=-|H|^{-1} \tilde{\chi}\left(\Delta\left(S_{p}(H)\right)\right.$. Moreover by Proposition 3.3, we have

$$
\widehat{f}_{p^{\prime}}(H)= \begin{cases}\mu(1, H) & \text { if } H \text { is an elementary abelian } p \text {-group, } \\ 0 & \text { otherwise. }\end{cases}
$$

(4.2) EXAMPLE. More generally let $K$ be a subgroup of $G$ and $f$ the function defined in (1.4). Then by Proposition $3.3 \widehat{f}_{p^{\prime}}(H)$ is non-zero only if $H$ is conjugate to a subgroup of the form $P \rtimes S$ where $S$ is conjugate to $K$ and normalizes $P, P$ is an elementary abelian $p$-group and $O_{p}(P \rtimes S)=P$. If we assume that $K$ is not contained in any $p$-local subgroup of $G$, then $f$ vanishes on $p$-local subgroups, so that $f=f_{p^{\prime}}$. In contrast if we assume that $K$ is a $p$-local subgroup, then the condition $O_{p}(P \rtimes S)=P$ is never satisfied so that $\widehat{f}_{p^{\prime}}=0$ and hence $f_{p^{\prime}}=0$; thus $f=f_{p}$ in that case. In general however, as in (4.1), $f$ has a non-trivial decomposition.

We now consider the constant function $f=1$. By (1.5) we have $\widehat{f}=\phi$, where

$$
\phi(H)= \begin{cases}\phi(|H|) & \text { if } H \text { is cyclic, } \\ 0 & \text { otherwise. }\end{cases}
$$

Let $\chi_{p}$ (respectively $\chi_{p^{\prime}}$ ) be the characteristic function of the set of subgroups of order divisible by $p$ (respectively prime to $p$ ).

(4.3) PROPOSITION. $1=\chi_{p}+\chi_{p^{\prime}}$ is the decomposition of the constant function with respect to the direct sum of Theorem 3.1.

Proof. It is clear that $\chi_{p^{\prime}}$ vanishes on $p$-local subgroups, so that $\chi_{p^{\prime}} \in \mathcal{F}_{p^{\prime}}(G, \mathbb{Z})$. Thus we only have to prove that $\widehat{\chi}_{p}(H)=0$ if $O_{p}(H)=1$. If $p \nmid|H|$, then $\chi_{p}$ is zero on all subgroups of $H$ and so $\widehat{\chi}_{p}(H)=0$. If $p|| H \mid$, then $H$ is not cyclic $\bmod p$ since $O_{p}(H)=1$. We recall that a group $H$ is called cyclic mod $p$ if $H / O_{p}(H)$ is a cyclic group (necessarily of order prime to $p$ ). Therefore by a theorem of Conlon [Co], the primitive idempotent $e_{H}$ of the Burnside algebra $B(H)$ which corresponds to $H$ is mapped to zero in the Green ring $A(H)$ of $k H$-modules, where $k$ is an algebraically closed field of characteristic $p$. But by a result of Gluck [Gl], this idempotent is equal to

$$
e_{H}=|H|^{-1} \sum_{S \leq H} \mu(S, H)|S| H / S
$$

and so the image of $|H| e_{H}$ in $A(H)$ is

$$
0=\sum_{S \leq H} \mu(S, H)|S| \operatorname{Ind}_{S}^{H}(k)
$$

where $k$ denotes the trivial representation of $H$. Now we take the inner product of this sum with $k$, in the sense of Benson and Parker (see [Be, Section 2.4]). We obtain

$$
0=\sum_{S \leq H} \mu(S, H)|S|<\operatorname{Ind}_{S}^{H}(k), k>_{H}=\sum_{S \leq H} \mu(S, H)|S|<k, k>_{S} .
$$

But one of the definitions of the inner product (see [Be, 2.4.1]) asserts that $\langle k, k\rangle_{S}$ is the multiplicity of the projective cover $P_{k}$ of the trivial module $k$ as a direct summand of $k$. Since $P_{k}=k$ if $S$ has order prime to $p$, but $P_{k} \neq k$ if $p$ divides $|S|$, it follows that $\left\langle k, k>_{S}\right.$ is the characteristic function $\chi_{p^{\prime}}$ of the set of $p^{\prime}$-subgroups. Thus we have

$$
0=\sum_{S \leq H} \mu(S, H)|S| \chi_{p^{\prime}}(S)=\widehat{\chi}_{p^{\prime}}(H) .
$$

Now since $H$ is not cyclic $\bmod p$ (in particular not cyclic), $\phi(H)=0$. Therefore we obtain $\widehat{\chi}_{p}(H)=$ $\phi(H)-\widehat{\chi}_{p^{\prime}}(H)=0$, as required. 
(4.4) COROLLARY (Webb $[\mathrm{W} 1,8.2]$ ). If $p$ divides the order of $H$ then

$$
\widetilde{\chi}\left(\Delta\left(S_{p}(H)\right) / H\right)=0 .
$$

Proof. We apply the formula for computing the $p^{\prime}$-part of the constant function.

$$
\chi_{p^{\prime}}(H)=\sum_{\sigma \in\left[\Delta\left(S_{p}(H)\right) / H\right]}(-1)^{\operatorname{dim}(\sigma)+1}=-\tilde{\chi}\left(\Delta\left(S_{p}(H)\right) / H\right) .
$$

This proves the result since $\chi_{p^{\prime}}(H)=0$ if $p$ divides $|H|$.

(4.5) REMARK. Webb proved later a more general result, namely that $\Delta\left(S_{p}(H)\right) / H$ is mod $p$ acyclic $[\mathrm{W} 3,2.6]$, and he conjectures that in fact $\Delta\left(S_{p}(H)\right) / H$ is contractible. Note that Corollary 4.4 is in fact equivalent to Proposition 4.3: assuming Webb's result (4.4), we obtain that the $p^{\prime}$-part of the constant function 1 is

$$
-\widetilde{\chi}\left(\Delta\left(S_{p}(H)\right) / H\right)=\left\{\begin{array}{lll}
0 & \text { if } p|| H \mid, & \text { by (4.4), } \\
1 & \text { if } p \nmid|H|, & \text { trivially. }
\end{array}\right.
$$

Therefore $\chi_{p^{\prime}}$ is the $p^{\prime}$-part of the constant function 1 , proving (4.3).

(4.6) REMARK. We have seen in the proof of (4.3) that $\widehat{\chi}_{p^{\prime}}(H)=0$ if $H$ is not cyclic mod $p$. If $H$ is cyclic $\bmod p$, one can apply Corollary 3.5 to get

$$
\widehat{\chi}_{p^{\prime}}(H)=\left|O_{p}(H): C_{O_{p}(H)}(H)\right| \mu\left(\left(1, O_{p}(H)\right)^{H}\right) \phi\left(H / O_{p}(H)\right) .
$$

One can also deduce easily a formula for $\widehat{\chi}_{p}(H)$.

\section{Further decomposition indexed by $p$-subgroups}

The following result shows that another (maybe more common) way of defining p-locally determined functions implies $p$-local determination in our sense. We shall prove below that both approaches are in fact equivalent.

(5.1) PROPOSITION. Let $f \in \mathcal{F}(G, A)$. Assume that for each $H \leq G$, the evaluation $f(H)$ can be written

$$
f(H)=\sum_{Q \in\left[S_{p}(H) / H\right]} h_{Q}\left(N_{H}(Q)\right),
$$

where for each non-trivial $p$-subgroup $Q \in S_{p}(G), \quad h_{Q}$ is some function in $\mathcal{F}\left(N_{G}(Q), A\right)$ such that $h_{s_{Q}}\left({ }^{s} K\right)=h_{Q}(K)$ for each $s \in G$ and $K \leq N_{G}(Q)$. Then $f$ is $p$-locally determined.

Proof. Let $H$ be a subgroup such that $O_{p}(H)=1$. We must prove that $\widehat{f}(H)=0$. Since the family $\left(h_{Q}\right)$ is $G$-stable by assumption, we can sum over all $p$-subgroups rather than representatives.

$$
\begin{aligned}
\widehat{f}(H) & =\sum_{S \leq H} \mu(S, H)|S| f(S) \\
& =\sum_{S \leq H} \mu(S, H)|S| \sum_{Q \in S_{p}(S)}\left|S: N_{S}(Q)\right|^{-1} h_{Q}\left(N_{S}(Q)\right) \\
& =\sum_{S \leq H} \mu(S, H) \sum_{Q \in S_{p}(S)}\left|N_{S}(Q)\right| h_{Q}\left(N_{S}(Q)\right) \\
& =\sum_{Q \in S_{p}(H)} \sum_{S \geq Q} \mu(S, H)\left|N_{S}(Q)\right| h_{Q}\left(N_{S}(Q)\right) \\
& =\sum_{Q \in S_{p}(H)} \sum_{Q \leq K \leq N_{H}(Q)}|K| h_{Q}(K) \sum_{S \geq Q, N_{S}(Q)=K} \mu(S, H) .
\end{aligned}
$$


Note that $K$ is always a proper subgroup of $H$ because $Q$ is a normal $p$-subgroup of $K$ while $O_{p}(H)=1$ by assumption. Write $[K, H]$ for the the poset of subgroups $S$ such that $K \leq S \leq H$. For fixed $Q$ and $K$, we have

$$
\sum_{S \geq Q, N_{S}(Q)=K} \mu(S, H)=\sum_{\substack{S \in[K, H] \\ S \cap N_{H}(Q)=K}} \mu(S, H),
$$

and this is zero thanks to the next well-known lemma (and because $N_{H}(Q)<H$ ). As a result we obtain $\widehat{f}(H)=0$ as required.

(5.2) LEMMA. Let $\mathcal{L}$ be a finite lattice with unique smallest element $K$ and unique largest element $H$. Write $\wedge$ for the meet operation in $L$. If $X \in \mathcal{L}$ with $X<H$ then

$$
\sum_{\substack{S \in \mathcal{L} \\ S \wedge X=K}} \mu(S, H)=0
$$

Proof. This standard result can be found in [St, 3.9.3]. For a change we sketch a topological proof. First note that

$$
\sum_{\substack{S \in \mathcal{L} \\ S \wedge X=K}} \mu(S, H)=\sum_{S \in \mathcal{L}} \mu(S, H)-\sum_{\substack{S \in \mathcal{L} \\ S \wedge X \neq K}} \mu(S, H),
$$

and the first sum is zero by the definition of the Möbius function. We can assume that $K<X$ otherwise the second sum is zero because it runs over the empty set. It is well known [St, 3.8.6] that this second sum is equal to $-\widetilde{\chi}(\mathcal{P})$ where $\mathcal{P}$ is the poset of all $S \in \mathcal{L}$ such that $S<H$ and $S \wedge X \neq K$. Here $\widetilde{\chi}(\mathcal{P})=\chi(\mathcal{P})-1$ denotes the reduced Euler characteristic of $\mathcal{P}$. Thus it suffices to show that this poset $\mathcal{P}$ is contractible. But this follows immediately from the following contractions (see [Qu, 1.5] for details): $S \mapsto S \wedge X \mapsto X$. Note that $X \in \mathcal{P}$ since $X<H$ by assumption.

(5.3) REMARK. The proof of Proposition 5.1 also gives the value of $\widehat{f}(H)$ when $O_{p}(H) \neq 1$. Indeed with the notation of that proof, if $Q$ is not normal in $H$, then the contribution to the sum is zero by the same argument as in the proof, while if $Q \triangleleft H$, the contribution is

$$
\sum_{S \geq Q} \mu(S, H)|S| h_{Q}(S)=|Q| \widehat{\bar{h}}_{Q}(H / Q)
$$

where $\bar{h}_{Q}: S\left(N_{G}(Q) / Q\right) \rightarrow \mathbb{Q}$ is defined by $\bar{h}_{Q}(S / Q)=h_{Q}(S)$, and $\widehat{\bar{h}}_{Q}$ is the inversion of $\bar{h}_{Q}$ in the poset $S\left(N_{G}(Q) / Q\right)$. Therefore we obtain

$$
\widehat{f}(H)=\sum_{\substack{Q \triangleleft H \\ Q \neq 1}}|Q| \widehat{\bar{h}}_{Q}(H / Q) .
$$

For a given function $f$, there are in general several possible functions $h_{Q}$ satisfying the hypothesis of Proposition 5.1. But, as we are now going to show, the extra condition that each function $\bar{h}_{Q}$ is a $p^{\prime}$-function (where $\left.\bar{h}_{Q}(K / Q)=h_{Q}(K)\right)$ insures uniqueness (as well as existence) of a decomposition of a $p$-locally determined function $f$. Our method consists in a generalization of the decomposition of Section 3 . 
For any $p$-subgroup $Q$ of $G$, let $\bar{N}_{G}(Q)=N_{G}(Q) / Q$. Consider the abelian group

$$
\left(\prod_{Q \in\{1\} \cup S_{p}(G)} \mathcal{F}_{p^{\prime}}\left(\bar{N}_{G}(Q), A\right)\right)^{G} \cong \prod_{Q \in\left[\{1\} \cup S_{p}(G) / G\right]} \mathcal{F}_{p^{\prime}}\left(\bar{N}_{G}(Q), A\right) .
$$

A family of functions $\left(\bar{h}_{Q}\right)_{Q \in\{1\} \cup S_{p}(G)}$ is $G$-stable if $\bar{h}_{s_{Q}}\left({ }^{g} K /{ }^{g} Q\right)=\bar{h}_{Q}(K / Q)$ for each $g \in G$ and $Q \leq K \leq N_{G}(Q)$. Since each $\bar{h}_{Q}$ is constant on conjugacy classes, it is $N_{G}(H)$-stable; thus the $G$-stable product of the left hand side is isomorphic to the product over representatives of the right hand side.

We define a $\mathbb{Z}$-linear homomorphism

$$
\Phi:\left(\prod_{Q \in\{1\} \cup S_{p}(G)} \mathcal{F}_{p^{\prime}}\left(\bar{N}_{G}(Q), A\right)\right)^{G} \longrightarrow \mathcal{F}(G, A)
$$

by mapping a $G$-stable family $\left(\bar{h}_{Q}\right)$ to the function $f$ defined by

$$
f(H)=\sum_{Q \in\left[\{1\} \cup S_{p}(H) / H\right]} \bar{h}_{Q}\left(N_{H}(Q) / Q\right) .
$$

Note that contrary to Proposition 5.1 we define the map only on the $p^{\prime}$-part of $\mathcal{F}\left(\bar{N}_{G}(Q), A\right)$ for each $Q$. Note also that the term indexed by $Q=1$ gives the $p^{\prime}$-part of $f$ because

$$
f(H)=\bar{h}_{1}(H)+\left(\sum_{Q \in\left[S_{p}(H) / H\right]} \bar{h}_{Q}\left(N_{H}(Q) / Q\right)\right),
$$

and the second term is $p$-locally determined by Proposition 5.1. Our main result is the following.

(5.4) THEOREM. The map

$$
\Phi:\left(\prod_{Q \in\{1\} \cup S_{p}(G)} \mathcal{F}_{p^{\prime}}\left(\bar{N}_{G}(Q), A\right)\right)^{G} \longrightarrow \mathcal{F}(G, A)
$$

is an isomorphism of abelian groups.

The main ingredient for the proof is an explicit formula for the inverse of $\Phi$, that is, as in Section 3, a formula for the projection onto each factor $\mathcal{F}_{p^{\prime}}\left(\bar{N}_{G}(Q), A\right)$, involving again Brown's complex. We define a $\mathbb{Z}$-linear map

$$
\begin{aligned}
\mathcal{F}(G, A) & \rightarrow \mathcal{F}_{p^{\prime}}\left(\bar{N}_{G}(Q), A\right) \quad ; \quad \bar{f}_{Q}(K / Q)=\sum_{\sigma \in\left[\Delta\left(S_{p}(K / Q)\right) /(K / Q)\right]}(-1)^{\operatorname{dim}(\sigma)+1} f\left(K_{\sigma}\right) . \\
f & \mapsto \bar{f}_{Q}
\end{aligned}
$$

Here $K_{\sigma}$ obviously contains $Q$ and $K_{\sigma} / Q$ is the stabilizer of $\sigma$ in $K / Q$. The map consists in restricting the function $f$ to the subgroups sandwiched between $Q$ and $N_{G}(Q)$, viewing the resulting function as defined on subgroups of $\bar{N}_{G}(Q)$, and then projecting onto the $p^{\prime}$-part of this function, using the explicit formula of Section 3. Thus the image of the map is indeed contained in $\mathcal{F}_{p^{\prime}}\left(\bar{N}_{G}(Q), A\right)$. Note that if $Q=1$, we recover the projection of Section 3 . Thus $\bar{f}_{1}=f_{p^{\prime}}$. Note also that the family of functions $\left(\bar{f}_{Q}\right)$ is $G$-stable. Thus the family of maps $f \mapsto \bar{f}_{Q}$ defines a $\mathbb{Z}$-linear map

$$
\Psi: \mathcal{F}(G, A) \longrightarrow\left(\prod_{Q \in\{1\} \cup S_{p}(G)} \mathcal{F}_{p^{\prime}}\left(\bar{N}_{G}(Q), A\right)\right)^{G} .
$$

Our first result asserts that $\Phi \Psi$ is the identity of $\mathcal{F}(G, A)$. 
(5.5) PROPOSITION. Let $f \in \mathcal{F}(G, A)$. For every subgroup $H$ of $G$, we have

$$
f(H)=\sum_{Q \in\left[\{1\} \cup S_{p}(H) / H\right]} \bar{f}_{Q}\left(\bar{N}_{H}(Q)\right) .
$$

Proof. We already know that $\bar{f}_{1}=f_{p^{\prime}}$, so we only have to deal with $f_{p}$. Now $f_{p}(H)$ is defined by a sum running over non-empty simplices $\sigma$ in $\Delta\left(S_{p}(H)\right)$. Writing $\sigma=\{Q\} \cup \tau$ with $\tau \in \Delta\left(S_{p}(H)_{>Q}\right)$, we obtain:

$$
\begin{aligned}
f_{p}(H) & =\sum_{\substack{\sigma \in\left[\Delta\left(S_{p}(H)\right) / H\right] \\
\sigma \neq \emptyset}}(-1)^{\operatorname{dim}(\sigma)} f\left(H_{\sigma}\right) \\
& =\sum_{Q \in\left[S_{p}(H) / H\right]} \sum_{\tau \in\left[\Delta\left(S_{p}(H)>Q\right) / N_{H}(Q)\right]}(-1)^{\operatorname{dim}(\tau)+1} f\left(N_{H}(Q) \cap H_{\tau}\right) \\
& =\sum_{Q \in\left[S_{p}(H) / H\right]}(-1)^{\operatorname{dim}(\tau)+1} f\left(N_{H}(Q)_{\tau}\right) \\
& =\sum_{Q \in\left[S_{p}(H) / H\right]} \bar{f}_{Q}\left(\bar{N}_{H}(Q)\right),
\end{aligned}
$$

using Lemma 2.3 to pass from $\Delta\left(S_{p}(H)_{>Q}\right)$ to $\Delta\left(S_{p}\left(\bar{N}_{H}(Q)\right)\right)$.

Specializing to $p$-locally determined functions, we obtain the existence of a decomposition as in Proposition 5.1.

(5.6) COROLLARY. If $f$ is $p$-locally determined, then for each $H \leq G$,

$$
f(H)=\sum_{Q \in\left[S_{p}(H) / H\right]} \bar{f}_{Q}\left(\bar{N}_{H}(Q)\right) .
$$

The fact that each $\bar{f}_{Q}$ is a $p^{\prime}$-function suffices to guarantee uniqueness, as we now prove. In other words we show that $\Psi \Phi$ is the identity.

(5.7) PROPOSITION. Let $f \in \mathcal{F}(G, A)$ be such that for each $H \leq G$,

$$
f(H)=\sum_{Q \in\left[\{1\} \cup S_{p}(H) / H\right]} \bar{h}_{Q}\left(N_{H}(Q) / Q\right)
$$

for some $G$-stable family of functions $\left(\bar{h}_{Q}\right) \in\left(\prod_{Q \in\{1\} \cup S_{p}(G)} \mathcal{F}_{p^{\prime}}\left(\bar{N}_{G}(Q), A\right)\right)^{G}$. Then $\bar{f}_{Q}=\bar{h}_{Q}$ for each $p$-subgroup $Q$.

Proof. We compute $\bar{f}_{P}$, where $P$ is a $p$-subgroup of $G$. Let $P \leq K \leq N_{G}(P)$.

$$
\begin{aligned}
& \bar{f}_{P}(K / P)=\sum_{\sigma \in\left[\Delta\left(S_{p}(K / P)\right) /(K / P)\right]}(-1)^{\operatorname{dim}(\sigma)+1} f\left(K_{\sigma}\right) \\
& =\sum_{\sigma \in\left[\Delta\left(S_{p}(K / P)\right) /(K / P)\right]}(-1)^{\operatorname{dim}(\sigma)+1} \sum_{Q \in\left[\{1\} \cup S_{p}\left(K_{\sigma}\right) / K_{\sigma}\right]} \bar{h}_{Q}\left(\bar{N}_{K_{\sigma}}(Q)\right) \\
& =\sum_{\sigma \in \Delta\left(S_{p}(K / P)\right)}\left|K / P: K_{\sigma} / P\right|^{-1}(-1)^{\operatorname{dim}(\sigma)+1} \sum_{Q \in\{1\} \cup S_{p}\left(K_{\sigma}\right)}\left|K_{\sigma}: N_{K_{\sigma}}(Q)\right|^{-1} \bar{h}_{Q}\left(\bar{N}_{K_{\sigma}}(Q)\right) \\
& =\sum_{Q \in\{1\} \cup S_{p}(K)}\left|K: N_{K}(Q)\right|^{-1} \sum_{\sigma \in \Delta\left(S_{p}(K / P)^{Q}\right)}(-1)^{\operatorname{dim}(\sigma)+1}\left|N_{K}(Q): N_{K_{\sigma}}(Q)\right|^{-1} \bar{h}_{Q}\left(\bar{N}_{K_{\sigma}}(Q)\right)
\end{aligned}
$$


If $Q \not \leq P$, we want to prove that the second sum is zero. We define a function $g \in \mathcal{F}\left(P N_{K}(Q) / P, A\right)$ by the formula $g(S / P)=\bar{h}_{Q}\left(S \cap N_{K}(Q) / Q\right)$. It is easy to see that $g$ is constant on $P N_{K}(Q)$-conjugacy classes of subgroups. Then we have $g\left(P N_{K_{\sigma}}(Q) / P\right)=\bar{h}_{Q}\left(N_{K_{\sigma}}(Q) / Q\right)$ and this allows to compute the sum.

$$
\begin{aligned}
& \sum_{\sigma \in \Delta\left(S_{p}(K / P) Q\right)}(-1)^{\operatorname{dim}(\sigma)+1}\left|N_{K}(Q): N_{K_{\sigma}}(Q)\right|^{-1} \bar{h}_{Q}\left(\bar{N}_{K_{\sigma}}(Q)\right) \\
= & \sum_{\sigma \in\left[\Delta\left(S_{p}(K / P)^{Q}\right) / N_{K}(Q)\right]}(-1)^{\operatorname{dim}(\sigma)+1} \bar{h}_{Q}\left(N_{K_{\sigma}}(Q) / Q\right) \\
= & \sum_{\sigma \in\left[\Delta\left(S_{p}(K / P)^{P Q / P}\right) /\left(P N_{K}(Q) / P\right)\right]}(-1)^{\operatorname{dim}(\sigma)+1} g\left(P N_{K_{\sigma}}(Q) / P\right) \\
= & \sum_{\sigma \in\left[\Delta\left(S_{p}(K / P)^{P Q / P}\right) / X\right]}(-1)^{\operatorname{dim}(\sigma)+1} g\left(X_{\sigma}\right)
\end{aligned}
$$

where $X=P N_{K}(Q) / P \leq N_{K}(P Q) / P=N_{K / P}(P Q / P)$. Since $Q \not \leq P$, the $p$-subgroup $P Q / P$ is non-trivial and Lemma 2.2 asserts that the sum above is zero.

Now if $Q<P$, we have $\bar{h}_{Q}\left(\bar{N}_{K_{\sigma}}(Q)\right)=0$. Indeed since $P$ is a $p$-group, the subgroup $R=N_{P}(Q)$ contains $Q$ properly and stabilizes $\sigma$ because $P$ does. Thus $R / Q \triangleleft \bar{N}_{K_{\sigma}}(Q)$ (because $P \triangleleft K$ implies $R \triangleleft N_{K}(Q)$ ), showing that $\bar{N}_{K_{\sigma}}(Q)$ is a $p$-local group. By assumption $\bar{h}_{Q}$ vanishes on $p$-local subgroups.

Now we are only left with $Q=P$, in which case $N_{K}(Q)=K$ and $N_{K_{\sigma}}(Q)=K_{\sigma}$. Therefore we have

$$
\bar{f}_{P}(K / P)=\sum_{\sigma \in \Delta\left(S_{p}(K / P)\right)}(-1)^{\operatorname{dim}(\sigma)+1}\left|K: K_{\sigma}\right|^{-1} \bar{h}_{P}\left(K_{\sigma} / P\right)=\bar{h}_{P}(K / P)
$$

because $\bar{h}_{P}$ vanishes on each $p$-local subgroup $K_{\sigma} / P$, except for $\sigma=\emptyset$.

By Propositions 5.5 and 5.7, the proof of Theorem 5.4 is now complete.

Up to now in this section, we have only decomposed the evaluation of a function $f$ at some subgroup $H$. We now wish to decompose the function itself, using a sum over $S_{p}(G) / G$ rather than a sum over $S_{p}(H) / H$ for each $H$. To this end it is convenient to introduce inflation and induction procedures. If $N$ is a normal subgroup of $K$ and $f \in \mathcal{F}(K / N, A)$, we define the inflation of $f$ by

$$
\operatorname{Inf}_{K / N}^{K} f \in \mathcal{F}(K, A) \quad ; \quad \operatorname{Inf}_{K / N}^{K} f(S)= \begin{cases}f(S / N) & \text { if } S \geq N \\ 0 & \text { otherwise. }\end{cases}
$$

If $K$ is a subgroup of $G$ and $f \in \mathcal{F}(K, A)$, we define the induction of $f$ by

$$
\operatorname{Ind}_{K}^{G} f \in \mathcal{F}(G, A) \quad ; \quad \operatorname{Ind}_{K}^{G} f(S)=\sum_{g \in[K \backslash G / S]} f\left(K \cap{ }^{g} S\right)
$$

It is easy to check that the resulting functions are well defined and constant on conjugacy classes of subgroups.

Applying $\operatorname{Inf}_{\bar{N}_{G}(Q)}^{N_{G}(Q)}$ and $\operatorname{Ind}_{N_{G}(Q)}^{G}$ to a function $f \in \mathcal{F}\left(\bar{N}_{G}(Q), A\right)$, the induction formula simplifies since $\operatorname{Inf}_{\bar{N}_{G}(Q)}^{N_{G}(Q)} f$ vanishes on subgroups not containing $Q$. Explicitely we have

$$
\operatorname{Ind}_{N_{G}(Q)}^{G} \operatorname{Inf}_{\bar{N}_{G}(Q)}^{N_{G}(Q)} f(H)=\sum_{g \in\left[N_{G}(Q) \backslash T_{G}(Q, H) / H\right]} \bar{f}_{Q}\left(N_{g_{H}}(Q) / Q\right)
$$

where $T_{G}(Q, H)=\left\{g \in G \mid Q \leq{ }^{g} H\right\}$. 
(5.8) COROLLARY. For any $f \in \mathcal{F}(G, A)$, we have a unique decomposition

$$
f=\sum_{Q \in\left[\{1\} \cup S_{p}(G) / G\right]} \operatorname{Ind}_{N_{G}(Q)}^{G} \operatorname{Inf}_{\bar{N}_{G}(Q)}^{N_{G}(Q)} \bar{f}_{Q} .
$$

In particular $f_{p}=\sum_{Q \in\left[S_{p}(G) / G\right]} \operatorname{Ind}_{N_{G}(Q)}^{G} \operatorname{Inf}_{\bar{N}_{G}(Q)}^{N_{G}(Q)} \bar{f}_{Q}$.

In other words

$$
\Phi=\sum_{Q \in\left[\{1\} \cup S_{p}(G) / G\right]} \operatorname{Ind}_{N_{G}(Q)}^{G} \operatorname{Inf}_{\bar{N}_{G}(Q)}^{N_{G}(Q)} .
$$

Proof. Since $\bar{f}_{1}=f_{p^{\prime}}$, we are left with the decomposition of $f_{p}$ and we apply Proposition 5.5 which gives the evaluation at a subgroup $H$. For every $p$-subgroup $Q$, we only have to group the $G$-conjugates of $Q$ contained in $H$.

$$
\begin{aligned}
f_{p}(H) & =\sum_{Q \in\left[S_{p}(H) / H\right]} \bar{f}_{Q}\left(\bar{N}_{H}(Q)\right) \\
& =\sum_{Q \in\left[S_{p}(G) / G\right]} \sum_{g \in\left[N_{G}(Q) \backslash T_{G}(Q, H) / H\right]} \bar{f}_{Q^{g}}\left(N_{H}\left(Q^{g}\right) / Q^{g}\right) \\
& =\sum_{Q \in\left[S_{p}(G) / G\right]} \sum_{g \in\left[N_{G}(Q) \backslash T_{G}(Q, H) / H\right]} \bar{f}_{Q}\left(N_{g_{H}}(Q) / Q\right) \\
& =\sum_{Q \in\left[S_{p}(G) / G\right]} \operatorname{Ind}_{N_{G}(Q)}^{G} \operatorname{Inf}_{\bar{N}_{G}(Q)}^{N_{G}(Q)} \bar{f}_{Q}(H) .
\end{aligned}
$$

Another way of expressing the results of this section is the following.

(5.9) COROLLARY. Let $E_{Q}$ be the $\mathbb{Z}$-linear endomorphism of $\mathcal{F}(G, A)$ defined by

$$
E_{Q}: f \mapsto \operatorname{Ind}_{N_{G}(Q)}^{G} \operatorname{Inf}_{\bar{N}_{G}(Q)}^{N_{G}(Q)} \bar{f}_{Q}
$$

Then $\left(E_{Q}\right)_{Q \in\left[\{1\} \cup S_{p}(G) / G\right]}$ is a family of orthogonal idempotents and their sum is the identity.

(5.10) REMARK. Write $f_{Q}=\operatorname{Ind}_{N_{G}(Q)}^{G} \operatorname{Inf}_{\bar{N}_{G}(Q)}^{N_{G}(Q)} \bar{f}_{Q}$ for simplicity. Then the inversion of $f_{Q}$ is easy to obtain in terms of the inversion of $\bar{f}_{Q}$ (which in turn can also be dealt with thanks to Proposition 3.3, since by definition $\bar{f}_{Q}$ is the $p^{\prime}$-part of the restriction of $f$ ). One obtains

$$
\widehat{f}_{Q}(S)=|Q| \sum_{\substack{R \triangleleft S \\ R=G Q}} \widehat{\bar{f}}_{Q}(S / R) .
$$

In particular $\widehat{f}_{Q}(S)=0$ unless $S \in \mathcal{X}_{Q}$ where $\mathcal{X}_{Q}$ is the conjugacy closure of the family of subgroups sandwiched between $Q$ and $N_{G}(Q)$. Thus $f_{Q}$ is $\mathcal{X}_{Q}$-determined. One can also restrict the family $\mathcal{X}_{Q}$ by using information about the vanishing of $\widehat{f}$. 


\section{Alperin's conjecture}

We consider several functions in $\mathcal{F}(G, \mathbb{Z})$ which all count irreducible representations of various kind. First $k$ and $\ell$ are the functions defined in the introduction.

For later use, we compute $k_{p^{\prime}}(H)$ and $\ell_{p^{\prime}}(H)$. Recall from the introduction that $\mathcal{A}$ denotes the class of groups $S$ such that $O_{p}(S)$ is elementary abelian and $S / O_{p}(S)$ is the direct product of (at most) two cyclic $p^{\prime}$-groups.

(6.1) LEMMA. Let $H$ be a subgroup of $G$.

(a) $k_{p^{\prime}}(H)=\ell_{p^{\prime}}(H)$. In other words $k-\ell$ is $p$-locally determined.

(b) $\quad k_{p^{\prime}}(H)=\frac{1}{|H|} \sum_{S \leq H, S \in \mathcal{A}} \mu\left(\left(1, O_{p}(S)\right)^{S}\right)\left|O_{p}(S): C_{O_{p}(S)}(S)\right| \phi_{2}\left(S / O_{p}(S)\right)$.

(c) $k_{p^{\prime}}(H)=\frac{1}{|H|} \sum_{S \leq H}\left(-\tilde{\chi}\left(\Delta\left(S_{p}(H)\right)^{S}\right)\right) \phi_{2}(S)=\frac{1}{|H|} \sum_{O_{p}(S)=1}\left(-\tilde{\chi}\left(\Delta\left(S_{p}(H)\right)^{S}\right)\right) \phi_{2}(S)$.

(d) $k_{p^{\prime}}(H)=\sum_{x}\left(-\tilde{\chi}\left(\Delta\left(S_{p}(H)\right)^{x} / C_{H}(x)\right)\right)$, where the sum runs over representatives of the conjugacy classes of $p$-regular elements of $H$.

Proof. (a) It is proved in [W3, 2.3.2] as well as in [KR, 4.5] that

$$
\sum_{\sigma \in\left[\Delta\left(S_{p}(H)\right) / H\right]}(-1)^{\operatorname{dim}(\sigma)}\left(k\left(H_{\sigma}\right)-\ell\left(H_{\sigma}\right)\right)=0 .
$$

This means that $k_{p^{\prime}}=\ell_{p^{\prime}}$. We now give another proof of the result. If $S$ is not the direct product of two cyclic groups, $\widehat{k}(S)=0=\widehat{\ell}(S)$; if $S$ is such a direct product and $O_{p}(S)=1$, then $S$ must be a $p^{\prime}$-group and $\widehat{k}(S)=\widehat{\ell}(S)$ by (1.8). Thus if $S$ is a subgroup such that $O_{p}(S)=1$, then $\widehat{k}(S)=\widehat{\ell}(S)$. This applies if $S$ is a complement of $O_{p}(H)$ for some $H$, and so by Proposition 3.3 we obtain $\widehat{k}_{p^{\prime}}=\widehat{\ell}_{p^{\prime}}$. Therefore $k_{p^{\prime}}=\ell_{p^{\prime}}$.

(b) By Corollary 3.5 and the fact that $\widehat{k}=\phi_{2}$ by (1.8), we have

$$
\widehat{k}_{p^{\prime}}(S)=\mu\left(\left(1, O_{p}(S)\right)^{S}\right)\left|O_{p}(S): C_{O_{p}(S)}(S)\right| \phi_{2}\left(S / O_{p}(S)\right),
$$

for every $S \leq G$. Moreover this is zero unless $S \in \mathcal{A}$. Summing over all $S \leq H$, the result follows from the definition of inversion of functions.

(c) We know that $\widehat{k}(S)=\phi_{2}(S)$ and we have seen in (3.9) that

$$
|H| k_{p^{\prime}}(H)=\sum_{S \leq H}\left(-\tilde{\chi}\left(\Delta\left(S_{p}(H)\right)^{S}\right)\right) \hat{k}(S)=\sum_{S \leq H, O_{p}(S)=1}\left(-\tilde{\chi}\left(\Delta\left(S_{p}(H)\right)^{S}\right)\right) \hat{k}(S) .
$$


(d) We compute further the right hand side of (c), changing its sign for simplicity and using the function $\ell$ instead of $k$ for convenience. We write $H_{p^{\prime}}$ for the set of elements of $H$ of order prime to $p$ and we use Example (1.8).

$$
\begin{aligned}
-|H| k_{p^{\prime}}(H) & =-|H| \ell_{p^{\prime}}(H)=\sum_{S \leq H} \tilde{\chi}\left(\Delta\left(S_{p}(H)\right)^{S}\right) \widehat{\ell}(S) \\
& =\sum_{\substack{x \in H_{p^{\prime}}, y \in H \\
x y=y x}} \tilde{\chi}\left(\Delta\left(S_{p}(H)\right)^{<x, y>}\right) \\
& =\sum_{x \in H_{p^{\prime}}} \sum_{y \in C_{H}(x)} \tilde{\chi}\left(\left(\Delta\left(S_{p}(H)\right)^{x}\right)^{y}\right) \\
& =\sum_{x \in H_{p^{\prime}}}\left|C_{H}(x)\right| \tilde{\chi}\left(\Delta\left(S_{p}(H)\right)^{x} / C_{H}(x)\right),
\end{aligned}
$$

because by Burnside's lemma applied to the set $\Delta_{m}$ of $m$-simplices in $\Delta\left(S_{p}(H)\right)^{x}$, we have

$$
\sum_{y \in C_{H}(x)}\left|\Delta_{m}^{y}\right|=\left|C_{H}(x)\right|\left|\Delta_{m} / C_{H}(x)\right|,
$$

and the alternating sum of these numbers gives $\left|C_{H}(x)\right| \tilde{\chi}\left(\Delta\left(S_{p}(H)\right)^{x} / C_{H}(x)\right)$. Finally summing only over representatives of $H$-conjugacy classes in $H_{p^{\prime}}$, we obtain

$$
-|H| k_{p^{\prime}}(H)=|H| \sum_{x} \tilde{\chi}\left(\Delta\left(S_{p}(H)\right)^{x} / C_{H}(x)\right)
$$

as required.

We now consider the function $z$ defined in the introduction. It is well known that a $p$-local group $S$ cannot have a representation in characteristic $p$ which is both simple and projective; thus $z(S)=0$. In other words $z \in \mathcal{F}_{p^{\prime}}(G, \mathbb{Z})$.

We also define $n p(H)=\ell(H)-z(H)$ (the number of non-projective irreducible $p$-modular characters of $H$ ) and $n d(H)=k(H)-z(H)$ (the number of ordinary irreducible characters of $H$ of dimension not divisible by $\left.|G|_{p}\right)$. Note that both $n p$ and $n d$ vanish on groups of order prime to $p$.

ALPERIN'S CONJECTURE [Al]. For every finite group $H$,

$$
\ell(H)=\sum_{Q \in\left[\{1\} \cup S_{p}(H) / H\right]} z\left(N_{H}(Q) / Q\right),
$$

or in other words

$$
n p(H)=\sum_{Q \in\left[S_{p}(H) / H\right]} z\left(N_{H}(Q) / Q\right) .
$$

If we allow $H$ to run over all subgroups of a given finite group $G$, we see that by Proposition 5.7, the above decomposition of $\ell$ should be the decomposition of Section 5. This is essentially the result of Knörr and Robinson $[\mathrm{KR}]$ which we now recall in our language. Their result actually states the equivalence below just for the evaluation of the functions at $N_{G}(Q) / Q$ for each $Q$, but as we consider functions, we have to work with the evaluations at each subgroup of $N_{G}(Q) / Q$. 
(6.2) THEOREM (Knörr - Robinson [KR]). The following conditions on a finite group $G$ are equivalent.

(1) For every $p$-subgroup $Q$ of $G$, Alperin's conjecture holds for all subgroups of $N_{G}(Q) / Q$.

(2) For every $p$-subgroup $Q$ of $G$, the functions $\ell_{p^{\prime}}$ and $z$, defined on the set of subgroups of $N_{G}(Q) / Q$, are equal.

Proof. We provide a proof, using our approach. By the uniqueness of the decomposition of functions (Proposition 5.7) and the fact that $z$ is a $p^{\prime}$-function, Alperin's conjecture asserts precisely that for each $p$-subgroup $Q$, the $p^{\prime}$-function $\bar{\ell}_{Q} \in \mathcal{F}_{p^{\prime}}\left(N_{G}(Q) / Q, \mathbb{Z}\right)$ defined in Section 5 should be equal to $z$. But

$$
\begin{aligned}
\bar{\ell}_{Q}(K / Q) & =\sum_{\sigma \in\left[\Delta\left(S_{p}(K / Q)\right) /(K / Q)\right]}(-1)^{\operatorname{dim}(\sigma)+1} \ell\left(K_{\sigma}\right) \\
& =\sum_{\sigma \in\left[\Delta\left(S_{p}(K / Q)\right) /(K / Q)\right]}(-1)^{\operatorname{dim}(\sigma)+1} \ell\left(K_{\sigma} / Q\right)=\ell_{p^{\prime}}(K / Q),
\end{aligned}
$$

because it is well known that $\ell(H / Q)=\ell(H)$ whenever $Q$ is a normal $p$-subgroup of $H$. Therefore $\bar{\ell}_{Q}$ is equal to the function $\ell_{p^{\prime}}$ defined on subgroups of $N_{G}(Q) / Q$.

Thus Alperin's conjecture comes down to the conjecture that $\ell_{p^{\prime}}=z$. We now state several equivalent versions of this equation. The third, fifth, sixth and eighth statements correspond to the conjectures of the introduction.

(6.3) THEOREM. The following conditions on a finite group $G$ are equivalent.

(1) The function $k_{p^{\prime}}=\ell_{p^{\prime}}$ is equal to $z$.

(2) $\ell=z+n p$ is the decomposition of $\ell$ into its $p^{\prime}$-part and its $p$-part.

(3) The function $n p$ is $p$-locally determined.

(4) $k=z+n d$ is the decomposition of $k$ into its $p^{\prime}$-part and its $p$-part.

(5) The function $n d$ is $p$-locally determined.

(6) The function $z$ is $\mathcal{A}$-determined.

(7) (Knörr - Robinson $[K R]) \quad z(H)=\sum_{\sigma \in\left[\Delta\left(S_{p}(H)\right) / H\right]}(-1)^{\operatorname{dim}(\sigma)} \ell\left(H_{\sigma}\right)$ for every $H \leq G$.

(8) $z(H)=\frac{1}{|H|} \sum_{S \leq H, S \in \mathcal{A}} \mu\left(\left(1, O_{p}(S)\right)^{S}\right)\left|O_{p}(S): C_{O_{p}(S)}(S)\right| \phi_{2}\left(S / O_{p}(S)\right)$, for every $H \leq G$.

(9) $z(H)=\frac{1}{|H|} \sum_{S \leq H}\left(-\tilde{\chi}\left(\Delta\left(S_{p}(H)\right)^{S}\right)\right) \phi_{2}(S)$, for every $H \leq G$.

(10) (Bouc [unpublished]) $z(H)=\sum_{x}\left(-\tilde{\chi}\left(\Delta\left(S_{p}(H)\right)^{x} / C_{H}(x)\right)\right)$ for every $H \leq G$, where the sum runs over representatives of the conjugacy classes of $p$-regular elements of $H$.

Proof. It is clear that (1), (2) and (3) are equivalent. Since $\ell_{p^{\prime}}=k_{p^{\prime}}$ by Lemma 6.1 , it is also clear that (1), (4) and (5) are equivalent.

(1) $\Rightarrow(6)$. We know by Lemma $6.1(\mathrm{~b})$ that $k_{p^{\prime}}$ is $\mathcal{A}$-determined.

$(6) \Rightarrow(5)$. Let $H$ be such that $O_{p}(H)=1$. We have to prove that $\widehat{n d}(H)=0$. Note first that $H \in \mathcal{A}$ if and only if $H$ is the direct product of two cyclic groups of order prime to $p$. In that case the function $n d$ is zero on every subgroup of $H$, and therefore $\widehat{n d}(H)=0$. If now $H \notin \mathcal{A}$, then $\widehat{z}(H)=0$ by assumption, and since $H$ is not the direct product of two cyclic groups, $\widehat{k}(H)=0$. Therefore $\widehat{n d}(H)=\widehat{k}(H)-\widehat{z}(H)=0$.

$(1) \Leftrightarrow(7)$. The right hand side of (7) is equal to $\ell_{p^{\prime}}$ by definition.

$(1) \Leftrightarrow(8) \Leftrightarrow(9) \Leftrightarrow(10)$. This is clear by Lemma 6.1 . 


\section{Relationship with the Burnside ring}

Let $B(G)$ be the Burnside ring of the group $G$. As mentioned in Section 2, any function $f \in \mathcal{F}(G, \mathbb{Z})$ can be viewed as an element of the dual $B(G)^{*}=\operatorname{Hom}_{\mathbb{Z}}(B(G), \mathbb{Z})$. Explicitely, the evaluation of $f$ on a basis element $G / H$ of $B(G)$ is defined to be $f(G / H)=f(H)$. Making this identification, we want to know what are the submodules $\mathcal{F}_{p}(G, \mathbb{Z})$ and $\mathcal{F}_{p^{\prime}}(G, \mathbb{Z})$ of $B(G)^{*}=\mathcal{F}(G, \mathbb{Z})$.

First recall that for every subgroup $S$ we have a ring homomorphism $\phi_{S}: B(G) \rightarrow \mathbb{Z}$ defined on each $G$-set $X$ by $\phi(X)=\left|X^{S}\right|$, the number of $S$-fixed points in $X$. The product of all $\phi_{S}$ (with $S$ taken up to conjugation) is a ring homomorphism from $B(G)$ to a product of copies of $\mathbb{Z}$, which is injective with an image of finite index.

If $U$ is a subset of $B(G)$ and $V$ a subset of $B(G)^{*}$, we define

$$
\begin{aligned}
& U^{\perp}=\left\{f \in B(G)^{*} \mid f(u)=0 \text { for all } u \in U\right\}, \\
& V^{\perp}=\{x \in B(G) \mid v(x)=0 \text { for all } v \in V\} .
\end{aligned}
$$

Let $\mathcal{X}$ be a set of subgroups of $G$ closed under conjugation. Let $B_{\mathcal{X}}$ be the $\mathbb{Z}$-span of all $G / S$ where $S$ runs in $\mathcal{X}$ and let $K_{\mathcal{X}}$ be the intersection of all $\operatorname{Ker}\left(\phi_{S}\right)$ where $S$ runs in $\mathcal{X}$. We only work with the $\mathbb{Z}$-module structure of these subsets, but for completeness we note that $K_{\mathcal{X}}$ is an ideal of $B(G)$, whereas in general $B_{\mathcal{X}}$ is not (unless $\mathcal{X}$ is closed under taking subgroups, in which case $B_{\mathcal{X}}$ is the image of induction from subgroups in $\mathcal{X}$ and $K_{\mathcal{X}}$ is the kernel of restriction to subgroups in $\mathcal{X}$ ). Both submodules $B_{\mathcal{X}}$ and $K_{\mathcal{X}}$ are pure submodules of $B(G)$, because $B_{\mathcal{X}}$ is spanned by a subset of the basis of $B(G)$, while if $\phi_{S}(n \cdot x)=0$ for $0 \neq n \in \mathbb{Z}$ then obviously $\phi_{S}(x)=0$.

(7.1) LEMMA. For every family $\mathcal{X}$ of subgroups closed under conjugation, we have

$$
\begin{aligned}
& B_{\mathcal{X}}^{\perp}=\{f \in \mathcal{F}(G, \mathbb{Z}) \mid f(H)=0 \text { if } H \in \mathcal{X}\}, \\
& K_{\mathcal{X}}^{\perp}=\{f \in \mathcal{F}(G, \mathbb{Z}) \mid \widehat{f}(H)=0 \text { if } H \notin \mathcal{X}\} .
\end{aligned}
$$

In particular if $\mathcal{X}$ is the set of all $p$-local subgroups of $G$, then

$$
B \frac{\perp}{\mathcal{X}}=\mathcal{F}_{p^{\prime}}(G, \mathbb{Z}) \quad \text { and } \quad K_{\mathcal{X}}^{\perp}=\mathcal{F}_{p}(G, \mathbb{Z}) .
$$

Proof. The first equality is clear since $f \in B_{\mathcal{X}}$ if and only if $f(G / S)=0$ for all $S \in \mathcal{X}$. For the second we first note that $\mathbb{Q} \otimes_{\mathbb{Z}} K_{\mathcal{X}}$ is spanned by the primitive idempotents $e_{H}$ where $H \notin \mathcal{X}$. Here $e_{H}$ is defined by the properties $\phi_{H}\left(e_{H}\right)=1$ and $\phi_{S}\left(e_{H}\right)=0$ if $S$ is not conjugate to $H$. Extending our functions to $\mathbb{Q} \otimes_{\mathbb{Z}} B(G)$, we see that $f \in K_{\mathcal{X}}$ if and only if $f\left(e_{H}\right)=0$ for all $H \notin \mathcal{X}$. But by a result of Gluck [Gl], we have

$$
e_{H}=\frac{1}{\left|N_{G}(H)\right|} \sum_{S \leq H} \mu(S, H)|S| G / S .
$$

It follows that $f \in K_{\mathcal{X}}^{\perp}$ if and only if $\sum_{S \leq H} \mu(S, H)|S| f(G / S)=0$ for all $H \notin \mathcal{X}$. This condition is exactly $\widehat{f}(H)=0$ for all $H \notin \mathcal{X}$.

It is easy to see that $B_{\mathcal{X}} \cap K_{\mathcal{X}}=0$ and that the dimensions of $\mathbb{Q} \otimes_{\mathbb{Z}} B_{\mathcal{X}}$ and $\mathbb{Q} \otimes_{\mathbb{Z}} K_{\mathcal{X}}$ add up to $\operatorname{dim}_{\mathbb{Q}}\left(\mathbb{Q} \otimes_{\mathbb{Z}} B(G)\right)$. Therefore

$$
\left(\mathbb{Q} \otimes_{\mathbb{Z}} B \mathcal{X}\right) \oplus\left(\mathbb{Q} \otimes_{\mathbb{Z}} K_{\mathcal{X}}\right)=\mathbb{Q} \otimes_{\mathbb{Z}} B(G),
$$

but in general $B_{\mathcal{X}} \oplus K_{\mathcal{X}}$ is a proper subgroup of $B(G)$ (of finite index). If for instance $\mathcal{X}$ is closed under taking subgroups, then both $B_{\mathcal{X}}$ and $K_{\mathcal{X}}$ are ideals, so correspond to idempotents of $\mathbb{Q} \otimes_{\mathbb{Z}} B(G)$; but if $G$ is soluble, it is a result of Dress [Dr] that no such idempotent lies in $B(G)$ (provided $\mathcal{X}$ is non-empty and proper). It is a rather special property of the family of all $p$-local subgroups that we do have such a decomposition over $\mathbb{Z}$. The result is equivalent to Theorem 3.1. 
(7.2) PROPOSITION. Let $\mathcal{X}$ be the family of all $p$-local subgroups of $G$. Then

$$
B_{\mathcal{X}} \oplus K_{\mathcal{X}}=B(G) .
$$

Proof. By Lemma 7.1, Theorem 3.1 asserts that $B_{\mathcal{X}}^{\perp} \oplus K_{\mathcal{X}}^{\perp}=B(G)^{*}$. Therefore $B_{\mathcal{X}}^{\perp \perp} \oplus K_{\mathcal{X}}^{\perp \perp}=B(G)$. But $B_{\mathcal{X}} \subseteq B_{\mathcal{X}}^{\perp \perp}$ and both are pure submodules of the same rank. Thus $B_{\mathcal{X}}=B_{\mathcal{X}}^{\perp \perp}$, and similarly $K_{\mathcal{X}}=K_{\mathcal{X}}^{\perp \perp}$.

Now we want to translate in the Burnside ring the decomposition of Section 5. It turns out that this provides a new proof of a result of Bouc. For a suitable set $\mathcal{F}$ of subgroups of $G$, Bouc [Bo] defines two families $\left(\phi_{P}^{G}\right)$ and $\left(\psi_{P}^{G}\right)$ of idempotent endomorphisms of $B(G)$ where $P$ runs in $\mathcal{F}$ up to conjugation. When $\mathcal{F}=\{1\} \cup S_{p}(G)$, he mentions [Bo. 1.7.4] that $\phi_{P}^{G}=\psi_{P}^{G}$. Thus we only consider $\psi_{P}^{G}$. For $P=1$, the definition is

$$
\begin{aligned}
\psi_{1}^{G}(G / H) & =-\operatorname{Ind}_{H}^{G}\left(\widetilde{\Lambda}_{H}\left(\Delta\left(S_{p}(H)\right)\right)\right) \\
& =-\operatorname{Ind}_{H}^{G}\left(\sum_{\sigma \in\left[\Delta\left(S_{p}(H)\right) / H\right]}(-1)^{\operatorname{dim}(\sigma)} H / H_{\sigma}\right) \\
& =\sum_{\sigma \in\left[\Delta\left(S_{p}(H)\right) / H\right]}(-1)^{\operatorname{dim}(\sigma)+1} G / H_{\sigma},
\end{aligned}
$$

and for arbitrary $P$, we have

$$
\psi_{P}^{G}=\operatorname{Ind}_{N_{G}(P)}^{G} \operatorname{Inf}_{N_{G}(P) / P}^{N_{G}(P)} \psi_{1}^{N_{G}(P) / P} \operatorname{Fix}_{N_{G}(P) / P}^{N_{G}(P)} \operatorname{Res}_{N_{G}(P)}^{G},
$$

where $\operatorname{Fix}_{N_{G}(P) / P}^{N_{G}(P)}: B\left(N_{G}(P)\right) \rightarrow B\left(N_{G}(P) / P\right)$ maps an $N_{G}(P)$-set $X$ to the subset $X^{P}$ of $P$-fixed points, and where Ind, Res and Inf are the induction, restriction and inflation maps.

Dualizing those maps, we have the following result.

(7.3) LEMMA. Let $H \leq G$ and let $P \triangleleft H$.

(a) $\left(\operatorname{Ind}_{H}^{G}\right)^{*}=\operatorname{Res}_{H}^{G}: \mathcal{F}(G, \mathbb{Z}) \rightarrow \mathcal{F}(H, \mathbb{Z})$.

(b) $\left(\operatorname{Res}_{H}^{G}\right)^{*}=\operatorname{Ind}_{H}^{G}: \mathcal{F}(H, \mathbb{Z}) \rightarrow \mathcal{F}(G, \mathbb{Z})$.

(c) $\left(\operatorname{Inf}_{H / P}^{H}\right)^{*}=\operatorname{Res}_{H / P}^{H}: \mathcal{F}(H, \mathbb{Z}) \rightarrow \mathcal{F}(H / P, \mathbb{Z})$.

(d) $\left(\operatorname{Fix}_{H / P}^{H}\right)^{*}=\operatorname{Inf}_{H / P}^{H}: \mathcal{F}(H / P, \mathbb{Z}) \rightarrow \mathcal{F}(H, \mathbb{Z})$.

(e) $\left(\psi_{P}^{G}\right)^{*}=E_{P}: \mathcal{F}(G, \mathbb{Z}) \rightarrow \mathcal{F}(G, \mathbb{Z})$ (as defined in Corollary 5.9).

Proof. (a) is clear because $f\left(\operatorname{Ind}_{H}^{G}(H / S)\right)=f(G / S)=f(S)=\operatorname{Res}_{H}^{G}(f)(H / S)$.

(b) follows from the Mackey decomposition for the restriction of $G$-sets:

$$
f\left(\operatorname{Res}_{H}^{G}(G / S)\right)=\sum_{g \in[H \backslash G / S]} f\left(H / H \cap{ }^{g} S\right)=\operatorname{Ind}_{H}^{G}(f)(G / S) .
$$

(c) is immediate.

(d) If $K \leq H$ does not contain $P$, then no conjugate of $K$ contains $P$. So $P$ does not stabilize any point of the $H$-set $H / K$ and $(H / K)^{P}=\emptyset$. If $P \leq K \leq H$, then $(H / K)^{P}=H / K$ (that is, $(H / P) /(K / P)$ as an $(H / P)$-set $)$. It follows that

$$
f\left(\operatorname{Fix}_{H / P}^{H}(H / K)\right)= \begin{cases}f((H / P) /(K / P)) & \text { if } P \leq K, \\ 0 & \text { otherwise. }\end{cases}
$$

This is precisely the definition of $\operatorname{Inf}_{H / P}^{H}(f)$.

(e) If $P=1$, it is immediate from the definition that $\left(\psi_{1}^{G}\right)^{*}$ is equal to the projection $f \mapsto f_{p^{\prime}}$. For arbitrary $P$, it follows from (a), (b), (c), (d) that $\left(\psi_{P}^{G}\right)^{*}$ is the composite of the restriction to $N_{G}(P) / P$, followed by the projection onto the $p^{\prime}$-part of functions, followed by inflation to $N_{G}(P)$ and induction to $G$. This is precisely the definition of the map $E_{P}$ of Corollary 5.9. 
Corollary 5.9 asserts that $\left(E_{P}\right)_{P \in\left[\{1\} \cup S_{p}(G) / G\right]}$ is a family of orthogonal idempotents whose sum is the identity. Dualizing this, we obtain Bouc's result.

(7.4) PROPOSITION (Bouc [Bo, Théorème 1]). $\quad\left(\psi_{P}\right)_{P \in\left[\{1\} \cup S_{p}(G) / G\right]}$ is a family of orthogonal idempotents whose sum is the identity.

\section{References}

[Ai] M. Aigner, Combinatorial Theory, Springer Verlag, Berlin, 1979.

[Al] J.L. Alperin, Weights for finite groups, Proc. Symp. Pure Math. 47 (1987), 369-379.

[Be] D. Benson, Modular Representation Theory: New Trends and Methods, Springer Lecture Notes 1081, 1984.

[Bo] S. Bouc, Projecteurs dans l'anneau de Burnside, projecteurs dans l'anneau de Green, modules de Steinberg généralisés, J. Algebra, to appear.

[Co] S.B. Conlon, Decompositions induced from the Burnside algebra, J. Algebra 10 (1968), 102-122.

[Dr] A. Dress, A characterisation of solvable groups, Math. Z. 110 (1969), 213-217.

[Gl] D. Gluck, Idempotent formula for the Burnside algebra with applications to the p-subgroup simplicial complex, Ill. J. Math. 25 (1981), 63-67.

[Hi] K.A. Hirsch, On a theorem of Burnside, Quart. J. Math. Oxford 1 (1950), 97-99.

[KR] R. Knörr, G.R. Robinson, Some remarks on a conjecture of Alperin, J. London Math. Soc. 39 (1989), 48-60.

[Qu] D. Quillen, Homotopy properties of the poset of non-trivial p-subgroups of a group, Adv. in Math. 28 (1978), 101-128.

[Ro] G.R. Robinson, The number of blocks with a given defect group, J. Algebra 84 (1983), 493-502.

[St] R. Stanley, Enumerative Combinatorics, Volume I, Wadsworth \& Brooks/Cole, Monterey California, 1986.

[Th] J. Thévenaz, Permutation representations arising from simplicial complexes, J. Combin. Theory Ser. A 46 (1987), 121-155.

[TW] J. Thévenaz, P.J. Webb, Homotopy equivalence of posets with a group action, J. Combin. Theory Ser. A, to appear.

[W1] P.J. Webb, A local method in group cohomology, Comment. Math. Helv. 62 (1987), 135-167.

[W2] P.J. Webb, Subgroup complexes, Proc. Symp. Pure Math. 47 (1987), 349-365.

[W3] P.J. Webb, A split exact sequence of Mackey functors, preprint, 1989. 